\title{
Psicooncología
}

ISSN: 1696-7240

\section{Propiedades Psicométricas del Instrumento de Evaluación de Funciona- lidad en Tratamiento para el Cáncer - Función Cognitiva (FACT-COG) en pacientes mexicanas con cáncer de mama.}

\author{
Luis Alberto Vázquez Rodríguez; ; Oscar Galindo Vazquez²; Enrique Bargalló Rocha3; \\ Abel Lerma; ${ }^{4}$ Paula Cabrera Galeana ${ }^{5}$; Karla Janeth Méndez Meneses ${ }^{6}$; Abelardo \\ Meneses García?
}

Recibido: 12 de noviembre de 2018 / Aceptado. 12 de mayo de 2019

Resumen: Introducción: Las pacientes con cáncer de mama presentan dentro de sus principales efectos en su calidad de vida durante o posterior al tratamiento deterioro cognitivo. Su detección y atención oportuna puede mejorar la funcionalidad de este grupo de pacientes. Objetivo: Determinar las propiedades psicométricas del Instrumento de Evaluación de Funcionalidad en Tratamiento Para el Cáncer - Función Cognitiva (FACTCOG) en una muestra de pacientes con cáncer de mama mexicanas. Método: Participaron 200 pacientes del Instituto Nacional de Cancerología, la edad promedio fue de 45.59 años. Se utilizó un diseño transversal no experimental. Instrumentos: FACT-COG, Escala de Ansiedad y Depresión Hospitalaria (HADS) y el Mini Examen del Estado Mental (MMSE). Análisis estadístico: Se realizó un modelo factorial exploratorio y confirmatorio, así como validez concurrente. Resultados: Se identificaron cuatro factores con 36 reactivos. Las consistencias internas de cada subescala tuvieron un valor de entre 0,98 a 0,95 , de la escala global 0,98 , que explicaron el $77,6 \%$ de la varianza. El análisis factorial confirmatorio presenta indicadores adecuados y del ajuste de la estructura del modelo (CFI, RMR y RMSEA) que indican un modelo equilibrado y parsimonioso. La validez, por medio de correlación con las medidas concurrentes, mostró resultados significativos ( $\mathrm{r}$ de Pearson de , 80 a -,55). Discusión y conclusión El FACT-COG en pacientes con cáncer de mama en población mexicana presentó adecuadas y robustas características psicométricas exploratorias y confirmatorias. La relevancia de los resultados obtenidos radica en que se trata de una población que puede llegar a requerir detección oportuna en afectaciones cognitivas durante su tratamiento.

Palabras clave: calidad de vida, cáncer de mama, función cognitiva, México, propiedades psicométricas.

1 Luis Alberto Vázquez Rodríguez Universidad Latina.

E-mail: vonska@hotmail.com.

2 Oscar Galindo Vazquez. Servicio de Psicooncología, Instituto Nacional de Cancerología. INCan.

E-mail: psigalindo@yahoo.com.mx

3 Enrique Bargalló Rocha Departamento de tumores mamarios. Instituto Nacional de Cancerología. INCan.

E-mail: ebargallo@incan.edu.mx

4 Abel Lerma Instituto de Ciencias de la Salud (ICSa), Universidad Autónoma del Estado de Hidalgo. UAEH.

E-mail: aleta_44@yahoo.ca

5 Paula Cabrera Galeana. Departamento de tumores mamarios. Instituto Nacional de Cancerología. INCan.

E-mail: drapaulacabrera@gmail.com

6 Karla Janeth Méndez Meneses. Universidad Latina.

E-mail: liebe_janeth21@hotmail.com

7 Abelardo Meneses Garcia. Dirección general, Instituto Nacional de Cancerología. INCan.

E-mail: amenesesg@incan.edu.mx

* Dirección de correspondencia: Oscar Galindo Vázquez. Servicio de Psicooncología, Instituto Nacional de Cancerología, Calle Av. San Fernando No.22, Colonia. Sección XVI Ciudad de México, C.P. 14080.

E-mail: psigalindo@yahoo.com.mx 


\title{
[en] Psychometric properties of the Functional Evaluation Instrument for Cancer Treatment - Cognitive Function (FACT-COG) in Mexican patients with breast cancer.
}

\begin{abstract}
Introduction: Patients with breast cancer present within their main effects on their quality of life during or after treatment cognitive impairment. Its timely detection and attention can improve the functionality of this group of patients. Objective: To determine the psychometric properties of the Functionality Assessment Instrument in Cancer Treatment - Cognitive Function (FACT-COG) in a sample of Mexican breast cancer patients. Method: 200 patients from the National Institute of Cancerology participated; the average age was 45.59 years. A non-experimental transverse design was used. Instruments: FACT-COG, Anxiety and Hospital Depression Scale (HADS) and the Mini Mental State Examination (MMSE). Statistical analysis: An exploratory and confimatory factorial model was carried out as well as concurrent validity. Results: Four factors with 36 items were identified. The internal consistencies of each subscale had a value of between 0.98 to 0.95 , of the global scale 0.98 , which explained $77.6 \%$ of the variance. The confirmatory factor analysis presents adequate indicators and the adjustment of the structure of the model (CFI, RMR and RMSEA) that indicate a balanced and parsimonious model. Validity, through correlation with concurrent measures, showed significant results (Pearson's $r$ from .80 to -.55). Discussion and conclusion The FACT-COG in patients with breast cancer in the Mexican population presented adequate and robust exploratory and confirmatory psychometric characteristics. The relevance of the results obtained is that it is a population that may require timely detection of cognitive impairments during treatment.
\end{abstract}

Keywords: Quality of life, breast cancer, cognitive function, Mexico, patients.

Sumario: 1. Introducción 2. Método 3. Resultados 4. Discusión 5. Conclusiones 6. Referencias

Cómo citar: Vázquez Rodríguez LA, Galindo Vazquez O, Bargalló Rocha E, Lerma A, Cabrera Galeana P, Méndez Meneses KJ, Meneses Garcia A. Propiedades Psicométricas del Instrumento de Evaluación de Funcionalidad en Tratamiento para el Cáncer - Función Cognitiva (FACT-COG) en pacientes mexicanas con cáncer de mama. Psicooncología. 2019; 16: 357-373. doi: 10.5209/psic.65596

\section{Introducción}

El cáncer de mama representa el $16 \%$ de todos los tipos de cáncer en la mujer a nivel mundial (Organización Mundial de la Salud, 2017)(1) en México es el cáncer más prevalente en mujeres mexicanas con 27283 durante el año 2017(2). Entre los efectos secundarios tardíos y a largo plazo del diagnóstico y tratamiento está el deterioro cognitivo el cual se ha reconocido como una preocupación en las mujeres con cáncer de mama ${ }^{(3,4)}$.

La incidencia en este grupo de pacientes es de 17 a $75 \% \%^{(5,6)}$ y de manera específica, afecta de un 19 a $78 \%$ a pacientes con cáncer de mama en quimioterapia ${ }^{(7)}$. Las causas del deterioro cognitivo son complejas y pueden incluir estrés después del diagnóstico de cáncer, ansiedad, depresión, miedo a la recurrencia o los efectos del tratamiento ${ }^{(8-10)}$.

Se han informado daños neuropsicológicos y neurotóxicos en el paciente, según la dosis empleada de quimioterapia, la vía de administración y la vulnerabilidad genética ${ }^{(7)}$ además de que se han encontrado diferencias en la prevalencia de deterioro cognitivo de las pacientes con cáncer de mama que son tratadas con diferentes dosis de quimioterapia, se reporta que el $32 \%$ de las pacientes con altas dosis de quimioterapia presentan deterioro en sus funciones ejecutivas, el $17 \%$ con dosis moderadas presentan deterioro cognitivo y el $9 \%$ con bajas dosis presentan deterioro $\operatorname{cognitivo~}^{(11)}$. 
El deterioro cognitivo inducido por la quimioterapia tiene como consecuencia la presencia de afectaciones importantes para la ejecución de funciones básicas del organismo, los cuales tienen influencia en el aprendizaje, funciones ejecutivas, la formación de la percepción, la visión o interpretación de las personas sobre situaciones comunes ${ }^{(7)}$.

La percepción de problemas cognitivos en pacientes con cáncer de mama atribuido a su tratamiento quimioterapéutico es muy común, además de que éste se asocia con la evaluación objetiva de la función cognitiva, efectos en la calidad de vida, distrés psicológico, ansiedad y depresión ${ }^{(12,13)}$. Así mismo, se ha reportado que hay una correlación positiva mayor entre la percepción de la función cognitiva del paciente con sintomatología psicológica y fatiga, en comparación con los resultados de test neuropsicológicos y las funciones ejecutivas; ya que se reporta que las pacientes que fueron sometidas a tratamientos de quimioterapia, además de presentar un deterioro cognitivo objetivo, presentan un estado emocional negativo, es decir, perciben un deterioro por el tratamiento lo cual es la causa de la reacción emocional pero no en todas las pacientes el deterioro cognitivo es realmente como ellas lo perciben, en dichos casos es necesaria una intervención psicológica en vez de una neuropsicológica ${ }^{(12,14,15)}$.

De acuerdo con Skirbe et al. (2013) ${ }^{(16)}$ la prevalencia de la percepción del deterioro cognitivo en las pacientes con cáncer de mama con base en la edad, indica que el $8 \%$ tienen de 20 a 39 años, el $18 \%$ tiene entre 40 y 49 años, el $22 \%$ entre 50 y 59 años, el $28 \%$ entre 60 y 69 años siendo este último grupo el de mayor riesgo, el 18\% entre 70 y 79 años y el 6\% tienen más de 80 años de edad. Según la raza y grupo étnico las pacientes caucásicas tienen una prevalencia de $62 \%$, las afroamericanas $14 \%$, las hispánicas $6 \%$ y las asiáticas $12 \%$; los síntomas cognitivos de mayor prevalencia son problemas con la memoria $62 \%$, enlentecimiento mental $62 \%$, disminución de la concentración $46 \%$, fatiga $72 \%$, preocupación por problemas de memoria $50 \%$ y perdida por el interés de la vida en un $30 \%$.

El deterioro cognitivo inducido por terapias antitumorales representa una nueva línea de investigación en los últimos años, además de que hay pocas pruebas validadas para evaluar el efecto en la calidad de vida y funcionalidad de estos déficits, provocando que los oncólogos y psico-oncólogos no cuenten con los instrumentos estandarizados para evaluar estos cambios cognitivos asociados con el tratamiento y factores psicológicos como la depresión o ansiedad ${ }^{(17,18)}$.

Por lo que en los últimos años se ha vuelto relevante la evaluación y medición del impacto del cáncer y los tratamientos sobre la calidad de vida y funcionalidad cognitiva de las pacientes con cáncer de mama, así como la afectación a su vida diaria. En este contexto el Instrumento de Evaluación de Funcionalidad en Tratamiento para el Cáncer - Función Cognitiva (FACT-COG) desarrollado por Wagner et al. (2009)(19) es un instrumento muy utilizado a nivel mundial, ya que ha sido validado en diferentes poblaciones, consta de 37 reactivos, en formato auto-aplicable, tiene como constructos la funcionalidad y calidad de vida en relación a la función cognitiva y se utiliza para evaluar la percepción auto-reportada de los síntomas neuropsicológicos en relación con el tratamiento quimioterapéutico y como ha afectado a su calidad de vida.

Las validaciones del FACT-COG han sido realizadas con distintos diagnósticos de cáncer y con diferentes esquemas de tratamiento de quimioterapia, ya sea antes, durante o posterior al tratamiento según el tipo de cáncer, en la validación realizada por Cheung et al. (2013) ${ }^{(20)}$ en pacientes asiáticas se identificó una confiabilidad 
de 0,92 , que conservo los 4 factores originales, con una varianza de 74,2\%. En la validación en población francesa ${ }^{(21)}$ en una muestra heterogénea de pacientes con cáncer de mama, pulmón, mieloma, ovario, peritoneo, próstata y recto en quimioterapia, se obtuvo una confiabilidad de 0.93 y sus 4 factores originales, además de que tuvo correlaciones moderadas con la Escala de Depresión Hamilton (HDRS) y correlaciones bajas con el (MMSE). Por último, la validación del FACT-COG en Corea $^{(22)}$ mostro una consistencia interna de 0,94 , correlaciones estadísticamente significativas con sintomatología de depresión y otras medidas de calidad de vida, conservando los 4 factores originales.

Sin embargo, en población mexicana con cáncer no existía un instrumento validado que evalué la percepción del deterioro cognitivo en la calidad de vida por lo que el objetivo de este estudio fue validar el FACT-COG en un grupo de pacientes con cáncer de mama sometidas a tratamiento quimioterapéutico.

\section{Método}

El estudio fue transversal, no experimental. Esta investigación fue aprobada por los comités de investigación y ética en investigación del INCan.

\section{Criterios de participación}

Inclusión: Pacientes ambulatorios con diagnóstico de cáncer de mama en tratamiento quimioterapéutico con al menos 2 ciclos y pacientes en seguimiento que aceptaron participar de manera voluntaria.

Exclusión: Cuadros psiquiátricos definidos como patrones de comportamiento de significación clínica que aparecen asociados a un malestar emocional o físico de la persona, a una discapacidad y al deterioro en el funcionamiento cotidiano. Problemas auditivos definido como la perdida de la audición que causa dificultades para oír una conversación o sonidos fuertes. Problemas visuales severos definido como una pérdida de la visión o ceguera, una alteración en la agudeza (visión borrosa o poco nítida), en la discriminación de los colores o en la percepción de movimientos y formas (visión doble o diplopía).

Eliminación: Que durante el proceso de llenado del instrumento decida no seguir participando en el estudio.

\section{Procedimiento}

\section{Fase I. Estudio piloto del instrumento FACT-COG.}

El objetivo del estudio piloto fue identificar problemas en la forma y construcción de los reactivos adaptados a la población mexicana se realizó el piloteo para la validación con una muestra de 30 pacientes en la Unidad Funcional de Cáncer de Mama del Instituto Nacional de Cancerología, se evaluó si había dificultad, confusión, palabras difíciles o lenguaje ofensivo, además de cómo el participante pudiera reformular la pregunta en caso de que considerara algún problema con los reactivos del instrumento; también se evaluó la comprensión de las opciones de respuesta. 
Fase II. Obtención de las propiedades psicométricas del Instrumento de Evaluación de Funcionalidad en Tratamiento Para el Cáncer-Función Cognitiva (FACT-COG).

Las participantes en el estudio fueron invitadas a participar, se les explico de forma clara y sin ambigüedades el objetivo del estudio, la confidencialidad de sus datos, el procedimiento y los beneficios del estudio; además se les aclaró que si en cualquier momento durante el llenado de los instrumentos deseaban retirarse no habría ningún problema, dicha evaluación se realizó previa a la consulta de oncología médica de la participante en el consultorio asignado para este fin.

Se recabaron los siguientes datos sociodemográficos mediante entrevista: sexo, edad, estado civil y nivel educativo; del expediente se obtuvieron datos sobre su condición médica: diagnóstico médico, tiempo desde diagnóstico, condición de enfermedad, comorbilidad, antecedentes familiares de cáncer, estadio, tratamientos, numero ciclos de quimioterapia recibidos y periodo actual del tratamiento. Si durante el proceso de participación se identificaron niveles de angustia clínicamente significativa o reacción emocional se les dio a las participantes de forma impresa información sobre el servicio de psicooncología y se hicieron las interconsultas a dicho servicio.

\section{Instrumentos}

El Instrumento de Evaluación de Funcionalidad en Tratamiento para el Cáncer Función Cognitiva (FACT-COG) ${ }^{(19)}$ es un instrumento que evalúa la percepción auto-reportada del paciente de sus habilidades cognitivas y los efectos de estos cambios cognitivos en su funcionalidad y calidad de vida, la prueba ha demostrado una consistencia interna, $\alpha=0,96$ (sin validación en población mexicana).

Mini Examen del Estado Mental (MMSE) ${ }^{(23)}$ es un instrumento diseñado para medir el estado o función cognitiva, consta de 11 ítems, evalúa memoria, orientación en el tiempo y espacio, atención, registro de palabras, lenguaje y calculo. Con una consistencia interna de $\alpha=0,89$, validado en población mexicana ${ }^{(24)}$.

Escala hospitalaria de ansiedad y depresión (HADS) ${ }^{(25)}$ instrumento que tiene como constructos la ansiedad y depresión, es un instrumento auto aplicable, conformado por 12 ítems. Con una consistencia interna de $\alpha=0,86$, validado en población mexicana oncológica ${ }^{(26)}$.

\section{Análisis estadísticos}

Análisis exploratorio. Se obtuvieron los estadísticos de tendencia central de cada reactivo para conocer su distribución en las opciones de respuestas; se determinaron los grupos extremos (cuartil 25 y 75) y con cada reactivo se llevó a cabo una prueba $t$ de Student para muestras independientes para identificar la propiedad de discriminación de cada reactivo, aquellos que tuvieran una $p>0,05$ fueron eliminados de los siguientes procedimientos.

Se realizaron análisis de tablas cruzadas para conocer la correlación entre ítems, a continuación, se llevó a cabo el análisis factorial considerando un valor $>0,40$ por reactivo. Con los reactivos que superaron estos análisis se estimó el alfa de Cronbach. Finalmente se obtuvo a través de $\mathrm{r}$ de Pearson las correlaciones entre los factores del FACT-COG con los factores del HADS y correlación Spearman entre los factores del FACT-COG y el puntaje total del MMSE, para estimar la validez concurrente. 
Análisis confirmatorio. Con los resultados exploratorios, se evaluó el ajuste del modelo de 4 factores mediante un análisis factorial confirmatorio (AFC) usando el método de máxima verosimilitud que incluyó los pasos de identificación y especificación del modelo, estimación de parámetros estandarizados, (correlaciones $\mathrm{R}^{2}$, covarianzas, índices de modificación y proporciones críticas de las diferencias), y por último, evaluación del ajuste mediante la observación de límites aceptables de los estimadores, así como no colinealidad en las variables medidas. Se estimaron los siguientes índices: $\mathrm{X}^{2}$, razón $\mathrm{X}^{2} / \mathrm{gl}$, índice de bondad de ajuste (GFI) y sus complementos (AGFI, TLI), así como el índice de bondad de ajuste comparativo $(\mathrm{CFI})^{(27)}$, que es el mejor indicador para muestras iguales o superiores a 200, y por último la raíz cuadrada media del error de aproximación (RMSEA).

\section{Resultados}

La muestra se obtuvo por disponibilidad durante la consulta de la Unidad Funcional de Cáncer de Mama (UFCM) del Instituto Nacional de Cancerología. La muestra consistió en 200 mujeres con diagnóstico de cáncer de mama, las cuales tuvieron una edad promedio de 45,59, el 31,5\% contaba con bachillerato como máximo grado de estudios, casadas $(40,5 \%)$, amas de casa $(43,5 \%)$ o desempleadas $(31,0 \%)$, con estadio III $(34,5 \%)$ y estadio IV $(33,0 \%)$ Ver tabla 1 para la descripción de la muestra.

Tabla 1. Descripción de la muestra de 200 pacientes diagnosticadas con cáncer de mama.

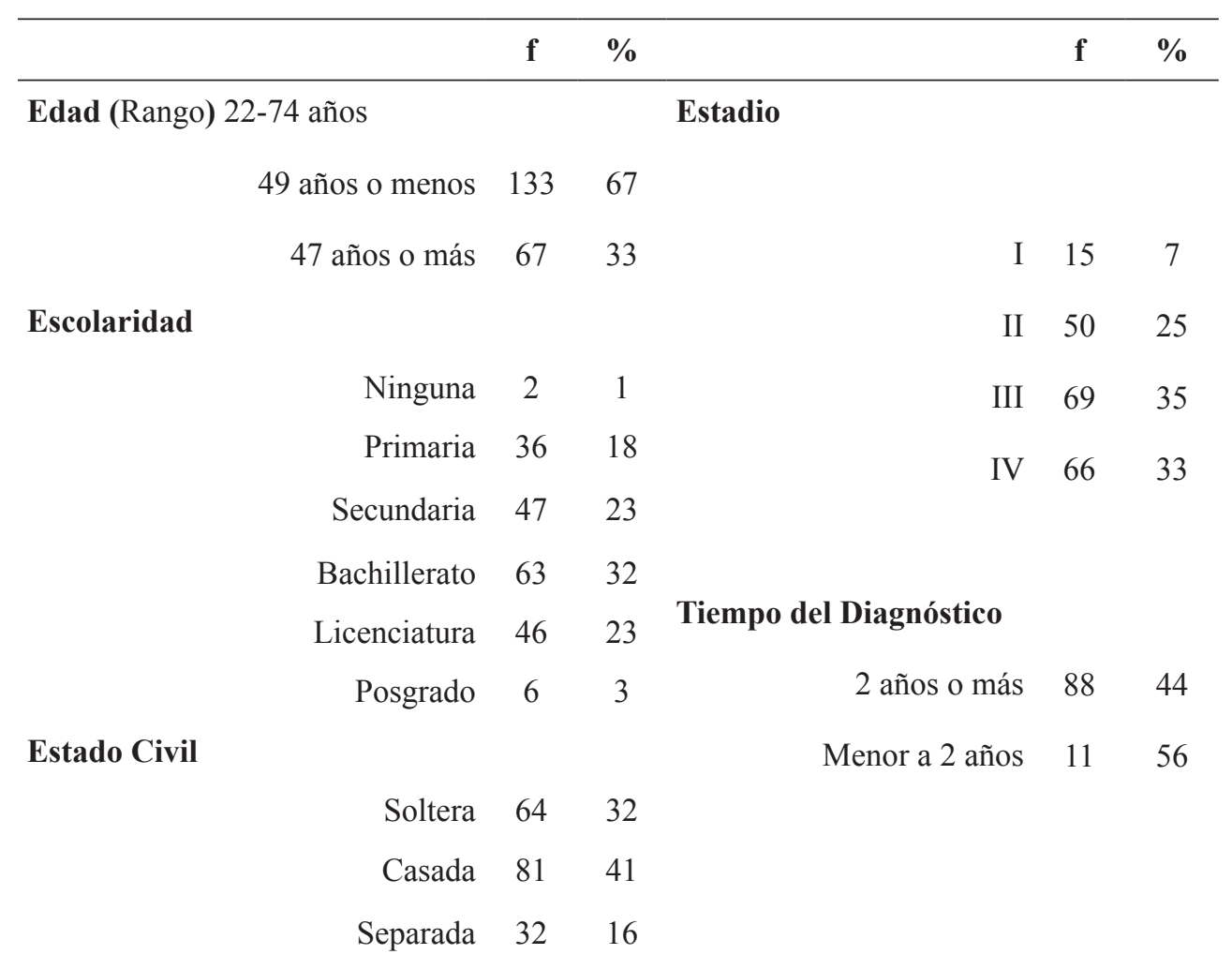




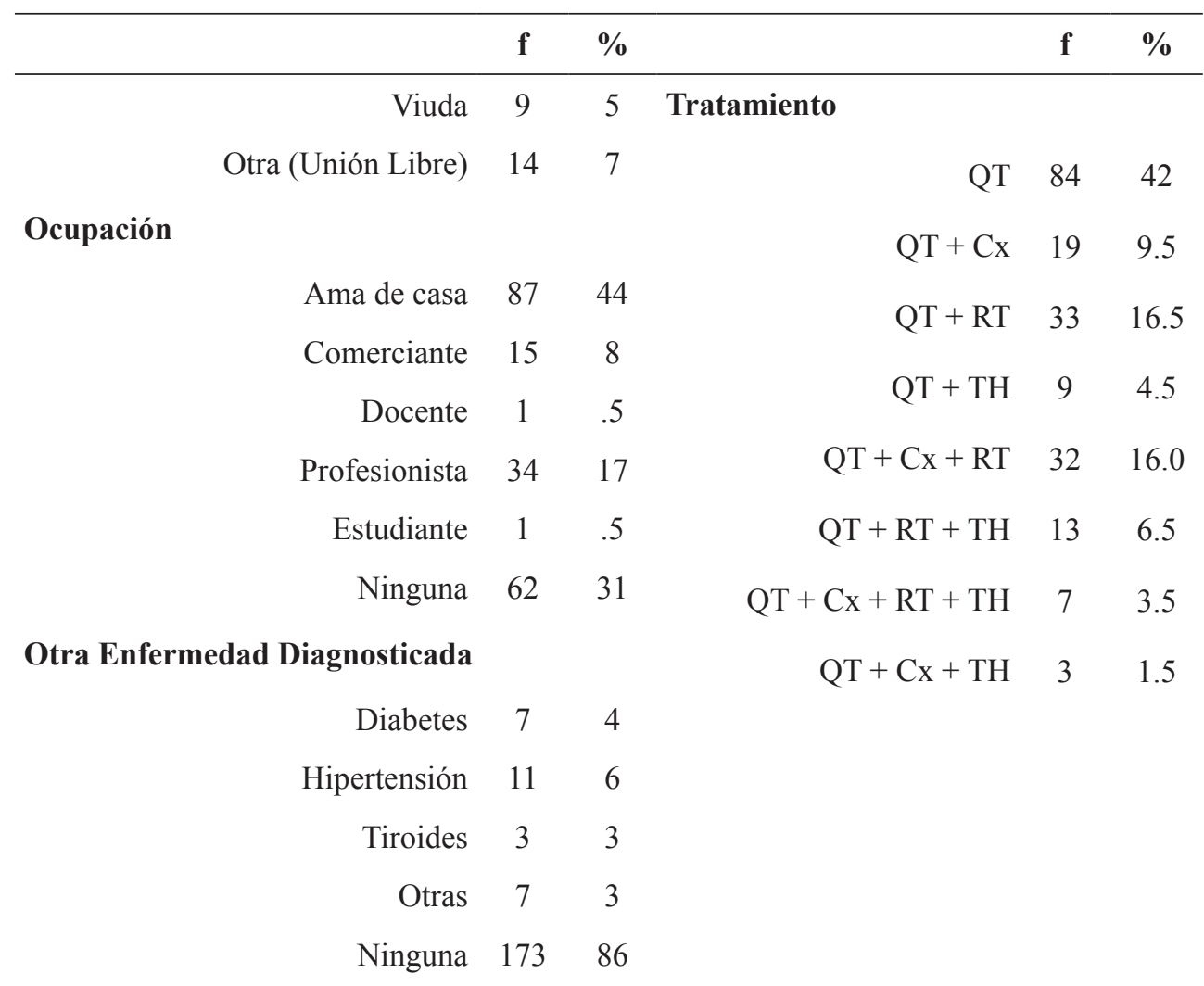

Atención Salud Mental

$\begin{array}{ccc}\text { Si } & 28 & 14 \\ \text { No } & 172 & 86\end{array}$

Nota: QT: Quimioterapia Cx: Cirugía RT: Radioterapia TH: Terapia Hormonal

Consistencia interna

El análisis de consistencia interna identificó 0,98 , de los 36 reactivos, se eliminó un reactivo que no obtuvo el mínimo de 0,40 . Se identificaron cuatro factores: Deficiencias cognitivas percibidas: $a=0,98$, Comentarios de Otros: $a=0.89$, Capacidades Cognitivas Percibidas: $a=0,95$ y Efectos en la Calidad de Vida: $a=0,95$.

Estructura factorial exploratoria

El análisis factorial exploratorio obtuvo en la prueba de esfericidad de Bartlett un valor $\mathrm{p}=0,001$ indicando no identidad de la matriz de correlación, mientras que el índice Kaiser-Meyer-Olkin (KMO) 0,96; para la extracción del modelo se utilizó la rotación Oblimin en la que se identificaron 4 factores que en conjunto explican el $77.6 \%$ de la varianza. Ver tabla 2 para la estructura factorial exploratoria. 
Tabla 2. Análisis Factorial de la validación del FACT-COG para pacientes con cáncer de mama en tratamiento quimioterapéutico.

Análisis Factorial

Escala Global a $=0,98$

Varianza Explicada $=77,6 \%$

Factor 1: Deficiencias Cognitivas Percibidas,

CogA1 He tenido dificultad para formar mis pensamientos,

CogA3 He pensado con lentitud

CogC7 He tenido dificultad para concentrarme

CogM9 He tenido dificultad para encontrar un lugar familiarizado

Carga

Factorial

Varianza

Explicada

$21,7 \%$

CogM10 He tenido dificultad para recordar donde pongo las cosas, como mis llaves o mi billetera,

CogM12 He tenido dificultad para recordar información nueva, como números de teléfonos o instrucciones sencillas,

CogV13 He tenido dificultad para recordar el nombre de un objeto cuando estoy hablando con alguien,

CogV15 He tenido dificultad para encontrar las palabras adecuadas para expresarme,

CogV16 He usado la palabra equivocada al referirme a un objeto,

CogV17b He tenido dificultad para expresar lo que quiero decir en mis conversaciones con los demás,

CogF19 He entrado en una habitación y se me ha olvidado lo que quería buscar o hacer allí,

CogF23 He tenido que esforzarme mucho para prestar atención o de lo contrario cometo

un error,

CogF24 Se me han olvidado los nombres de las personas al poco tiempo de haberse mencionado en la presentación,

CogF25 Mis reacciones ante las situaciones diarias han sido lentas, 
Análisis Factorial

Escala Global $\mathrm{a}=0,98$

Varianza Explicada $=77,6 \%$

CogC31He tenido que esforzarme más que de costumbre para seguir el hilo de lo que estaba haciendo,

CogC32 He pensado con más lentitud que de costumbre,

CogC33a He tenido que esforzarme más que de costumbre para expresarme con claridad,

$\mathrm{CogC} 33 \mathrm{c}$ He tenido que usar listas escritas con más frecuencia que de costumbre para que no se me olvidaran las cosas,

CogMT1 Tengo problemas para seguir el hilo de lo que estoy haciendo si me interrumpen,

CogMT2 Tengo problemas para alternar entre actividades distintas que requieren pensar,

Factor 2: Comentarios de Otros,

CogO1 Otras personas me han dicho que parecía tener problemas para recordar información,

$\mathrm{CogO} 2$ Otras personas me han dicho que parecía tener problemas para hablar con claridad,

CogO3 Otras personas me han dicho que parecía tener problemas para pensar con claridad,

$\mathrm{CogO} 4$ Otras personas me han dicho que parecía confundido,

Factor 3: Capacidades Cognitivas Percibidas,

CogPC1 He podido concentrarme

CogPV1 He podido encontrar las palabras que quería usar al conversar con los demás,

CogPM1 He podido recordar cosas, como

donde deje mis llaves o mi billetera,

CogPM2 He podido acordarme de hacer cosas, como tomar un medicamento o comprar algo que necesitaba,

$\begin{array}{lll}\text { Carga } & \text { Varianza } & \text { Alfa de } \\ \text { Factorial } & \text { Explicada } & \text { Cronbach }\end{array}$

, 854

, 847

, 770

,886

$8 \% \quad a=0,89$

, 855

, 728

$15,3 \% \quad a=0,95$ 


\begin{tabular}{|c|c|c|c|}
\hline \multicolumn{2}{|l|}{ Análisis Factorial } & \multirow{3}{*}{$\begin{array}{l}\text { Varianza } \\
\text { Explicada }\end{array}$} & \multirow{3}{*}{$\begin{array}{l}\text { Alfa de } \\
\text { Cronbach }\end{array}$} \\
\hline Escala Global a=0,98 & Carga & & \\
\hline Varianza Explicada $=77,6 \%$ & Factorial & & \\
\hline $\begin{array}{l}\text { CogPF1 Puedo prestar atención y seguir el } \\
\text { hilo de lo que estoy haciendo sin esforzarme } \\
\text { demasiado, }\end{array}$ & ,926 & & \\
\hline $\begin{array}{l}\text { CogPCH1 Mi agudeza mental ha sido la mis- } \\
\text { ma de siempre, }\end{array}$ & ,756 & & \\
\hline $\begin{array}{l}\mathrm{CogPCH} 2 \text { Mi memoria ha sido tan buena } \\
\text { como siempre, }\end{array}$ & ,612 & & \\
\hline $\begin{array}{l}\text { CogPMT1 Puedo alternar entre dos activida- } \\
\text { des que requieren pensar, }\end{array}$ & ,822 & & \\
\hline $\begin{array}{l}\text { CogPMT2 Puedo seguir el hilo de lo que es- } \\
\text { toy haciendo aunque me interrumpan, }\end{array}$ &, 747 & & \\
\hline Factor 4: Efectos en la Calidad de Vida, & & $15,2 \%$ & $\mathrm{a}=0,95$ \\
\hline CogQ35 Estos problemas me han angustiado, & ,856 & & \\
\hline $\begin{array}{l}\text { CogQ37 Estos problemas han afectado mi } \\
\text { capacidad para trabajar, }\end{array}$ & ,893 & & \\
\hline $\begin{array}{l}\text { CogQ38 Estos problemas han afectado mi } \\
\text { capacidad para hacer las cosas que disfruto, }\end{array}$ & ,860 & & \\
\hline $\begin{array}{l}\text { CogQ41 Estos problemas han afectado mi } \\
\text { calidad de vida, }\end{array}$ & ,791 & & \\
\hline
\end{tabular}

Estructura factorial confirmatoria

Se evaluó el ajuste del modelo de 4 factores obtenidos con el análisis exploratorio, mediante un análisis factorial confirmatorio (AFC) usando el método de máxima verosimilitud, con los siguientes pasos:

1) Identificación del modelo: Para el modelo analizado, de un total de 76 variables, se tuvieron 36 variables endógenas; hubo 40 variables no observadas (total de parámetros por estimar mediante el modelo), de las cuales 36 fueron de tipo exógeno, contra un total de elementos no redundantes de la matriz igual a 666. En razón de que los parámetros por estimar son claramente inferiores al total de elementos no redundantes de la matriz, concluimos que el modelo está sobre-identificado y puede identificarse, y la no existencia de correlaciones en los residuos indica que es recursivo.

2) Especificación del modelo: Basados en el análisis exploratorio de confiabilidad y factorial previamente presentado, se diseñó el modelo de la Figura 1 donde las variables dibujadas con circunferencias u óvalos representan variables latentes, mientras las variables observables mediante rectángulos. Los errores de medición y residuales se muestran como óvalos más pequeños. 
Figura 1.

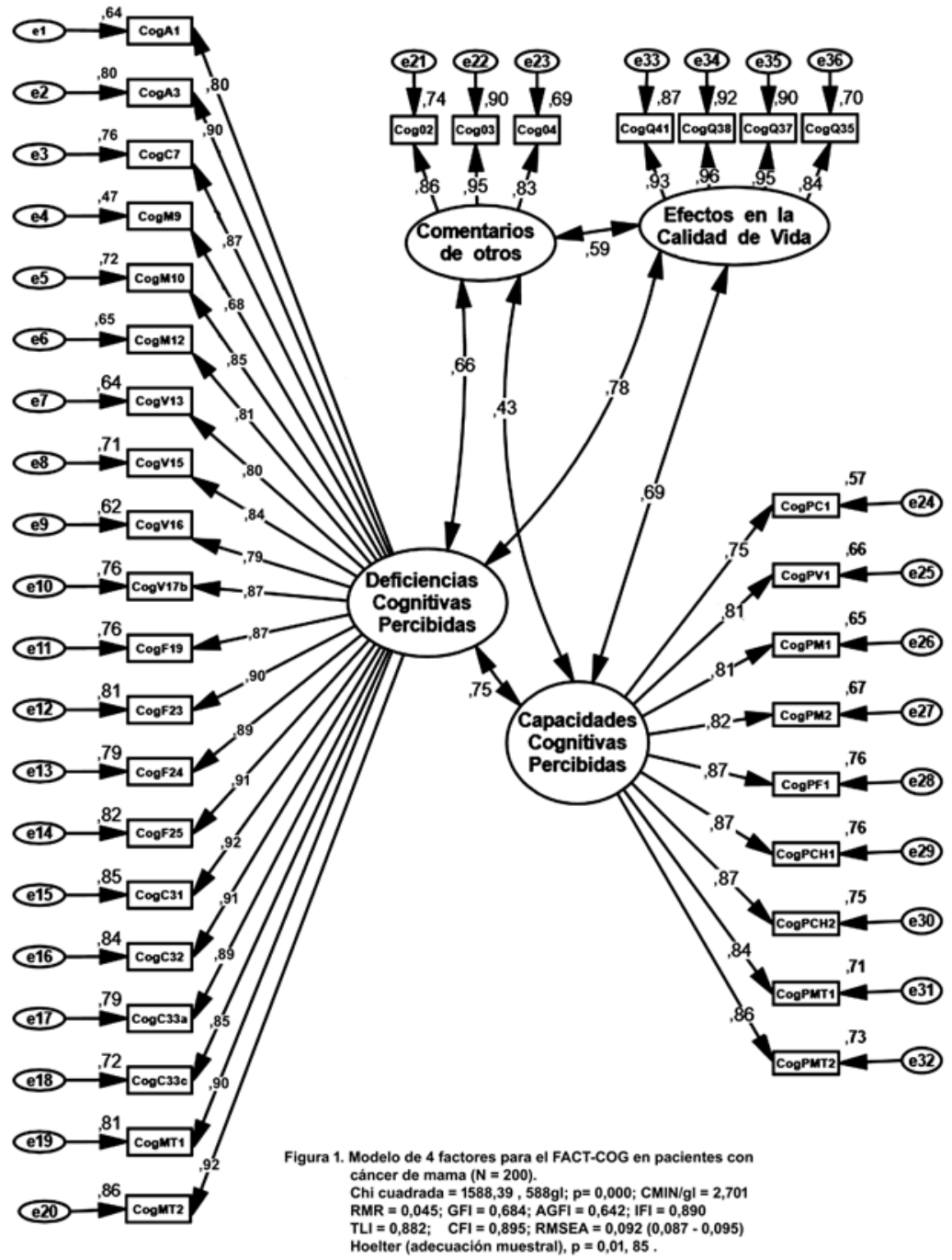

3) Estimación de parámetros: Mediante el programa AMOS se aplicó el método de máxima verosimilitud, con estimadores estandarizados, estimador $\mathrm{R}^{2}$ (correlaciones múltiples al cuadrado), covarianzas de los estimadores, índices por modificar y proporciones críticas para las diferencias.

4) Para la evaluación del ajuste (la muestra supera $n=199$ ), como primer paso se examinaron todos lo resultados para asegurar que los coeficientes estimados no excedieran los límites aceptables (que no hubiera varianzas de error negativas o no significativas, coeficientes estandarizados superiores a 1, o errores estándar excesivamente elevados relacionados con algún coeficiente estimado). 
No se observó colinealidad en las variables medidas, pues las correlaciones fueron menores a $0,3^{(28)}$ y tampoco puntajes extremos ni univariados ni multivariados ${ }^{(29)}$. Además, se observa asimetría excelente en todas las variables (valores no superiores $\mathrm{a} \pm 1,00)$.

5) La tabla 3 muestra que, en cuanto a las medidas absolutas del ajuste global, el valor chi cuadrada fue de 1429,15 ( $p=\leq 0,001,584$ g.1.), mientras que la razón chi cuadrada entre los grados de libertad $(\mathrm{CMIN} / \mathrm{DF}=2,45)$ es excelente, indicando que los errores en las varianzas y covarianzas del modelo son nulos y que está ajustado a muestras iguales o mayores a 200 datos según lo recomendado por expertos ${ }^{(30)}$.

Las medidas comparativas del ajuste global, como el índice comparativo (CFI), es casi ideal $(\mathrm{CFI}=0,907)$ así como el índice Tucker-Lewis, que toma en cuenta la complejidad del modelo (TLI $=0,900)$ indicando un ajuste aceptable a los datos ya que al menos el $90 \%$ de la covarianza en los datos puede ser reproducida por el modelo ${ }^{(30)}$.

Los índices de parsimonia ( $\mathrm{PCFI}=0,841$ ) están muy cercanos al valor óptimo (0.900), por lo que el modelo puede ser calificado como complejo ${ }^{(31)}$, como lo muestran aceptablemente los estadísticos de bondad de ajuste, $(\mathrm{GFI}=0,714, \mathrm{AGFI}=0,674$, PGFI $=0,626$ ), más la raíz del residuo cuadrático medio de aproximación (RMSEA $\left.=0,085, \mathrm{IC}^{95 \%}=0,080-0,091\right)$, que penaliza el incremento en la complejidad del modelo, e indica un aceptable ajuste a los datos al ser casi igual o menor a $0,08, \mathrm{y}$ que queda reafirmado por el valor en el error residual medio $(\mathrm{RMR}=0,047)$, pues éste cumple con el requisito de estar por abajo del valor límite, indicando error casi cero y ajuste casi perfecto (menor a 0,05$)^{(30)}$.

Tabla 3. Índices de bondad de ajuste del modelo confirmatorio resultante de 4 factores en pacientes con cáncer de mama $(\mathrm{n}=200)$,

\begin{tabular}{cccc}
\hline Estadístico & $\begin{array}{c}\text { Criterio } \\
\text { deseable }\end{array}$ & $\begin{array}{c}\text { Valor en el pre- } \\
\text { sente estudio }\end{array}$ & Interpretación \\
\hline $\begin{array}{c}\text { Ajuste absoluto } \\
\mathrm{X}^{2} / \mathrm{gl} \\
(\mathrm{CMIN} / \mathrm{gl})\end{array}$ & $\begin{array}{c}\text { Menor a } 2 \\
\text { o } 3\end{array}$ & $\begin{array}{c}1429,15 / 584 \mathrm{gl} \\
=2,447\end{array}$ & $\begin{array}{c}\text { Los errores del modelo son } \\
\text { nulos con la muestra utilizada y } \\
\text { el ajuste absoluto es excelente }\end{array}$ \\
$\begin{array}{c}\text { Índice de bondad de } \\
\text { ajuste } \\
(\text { GFI })\end{array}$ & $>0,90$ & GFI $=0,714$ & Ajuste bueno \\
$\begin{array}{c}\text { Preferente } \\
\text { ajuste comparativo } \\
\text { (CFI) }\end{array}$ & $\begin{array}{c}\text { Preferente } \\
>0,95\end{array}$ & CFI $=0,907$ & $\begin{array}{c}\text { Ajuste comparativo muy acep- } \\
\text { table }\end{array}$ \\
$\begin{array}{c}\text { Raíz del residuo cua- } \\
\text { drático medio (RMR) }\end{array}$ & $\begin{array}{c}\text { Próximo a } \\
\text { cero }\end{array}$ & RMR $=0,047$ & $\begin{array}{c}\text { Error del modelo próximo a } \\
\text { cero, casi perfecto ajuste del } \\
\text { modelo a los datos }\end{array}$ \\
$\begin{array}{c}\text { Raíz del residuo cua- } \\
\text { drático medio de apro- } \\
\text { ximación (RMSEA) }\end{array}$ & $\begin{array}{c}\text { Menor a } \\
0,08, \text { próxi- } \\
\text { mo a cero }\end{array}$ & $\begin{array}{c}\text { RMSEA }=0,085 \\
(0,080-0,091)\end{array}$ & $\begin{array}{c}\text { Error del modelo próximo a } \\
\text { cero, casi perfecto ajuste del } \\
\text { modelo a los datos }\end{array}$ \\
\hline
\end{tabular}




\section{Validez concurrente}

Se identificaron correlaciones altas y moderadas entre los factores del FACT-COG con los factores del HADS ( $\mathrm{r}$ de Pearson de 0,80 a $-0,55, p<0,05)$ y asociaciones bajas entre el FACT-COG y el MMSE ( $\mathrm{r}$ de Spearman de 0,48 a $0,40, p<0,05$ ). Ver tabla 4 para ver validez concurrente.

Tabla 4. Correlación entre FACT-COG con HADS y MMSE.

\begin{tabular}{cccccc}
\hline & $\begin{array}{c}\text { Factor 1. } \\
\text { Deterioro } \\
\text { Cognitivo } \\
\text { Percibido }\end{array}$ & $\begin{array}{c}\text { Factor 2. } \\
\text { Comentarios } \\
\text { de Otros }\end{array}$ & $\begin{array}{c}\text { Factor 3. } \\
\text { Capacidades } \\
\text { Cognitivas } \\
\text { Percibidas }\end{array}$ & $\begin{array}{c}\text { Factor 4. } \\
\text { Efectos en la } \\
\text { Calidad de } \\
\text { Vida }\end{array}$ & $\begin{array}{c}\text { Puntuación } \\
\text { Total FACT- } \\
\text { COG }\end{array}$ \\
\hline $\begin{array}{c}\text { Factor 1. } \\
\text { Ansiedad }\end{array}$ &, $697^{* *}$ &, $562^{* *}$ &,$- 553^{* *}$ &, $804^{* *}$ & \\
$\begin{array}{c}\text { Factor2. } \\
\text { Depresión }\end{array}$ &, $592^{* *}$ &, $434^{* *}$ &,$- 558^{* *}$ &, $712^{* *}$ & \\
$\begin{array}{c}\text { Puntuación } \\
\text { Total MMSE. }\end{array}$ &, $483^{* *}$ &, $425^{* *}$ &, $400^{* *}$ &, $473^{* *}$ &, $482^{* *}$ \\
\hline
\end{tabular}

\section{Discusión}

Los análisis psicométricos realizados para la validación del Instrumento de Evaluación de Funcionalidad en Tratamiento para el Cáncer- Función Cognitiva $\left(\right.$ FACT-COG) ${ }^{(19)}$ en pacientes con cáncer de mama sometidas a tratamiento quimioterapéutico, presenta una estructura factorial similar a la original y a las validaciones en otros países, la versión de este estudio mantuvo los 4 factores originales que son "Deterioro Cognitivo Percibido" (DCP), "Comentarios de Otros" (CO), "Habilidades Cognitivas Percibidas" (HCP) e "Impacto en la Calidad de Vida" (ICV) con una varianza explicada de 77,6\% y un total de 36 ítems.

El FACT-COG en este estudio mostró una consistencia interna con alfa de Cronbach de 0,98 similar a la del instrumento original que mostró un valor de 0,96, la validez fue estimada a partir de la correlación con la HADS que tienen como constructos la ansiedad y depresión, la cual mostró correlaciones altas y moderadas con los cuatro factores del FACT-COG, el constructo que tuvo más correlaciones altas fue ansiedad la cual mostró que a mayor deterioro cognitivo percibido por la paciente aumentaba la ansiedad, a mayor comentarios de otro aumentaba moderadamente, a mayor percepción de capacidades cognitivas percibidas decrementaba y a mayor impacto en la calidad de vida la correlación llego a ser la más alta en comparación con los demás constructos, muy similar a la escala original.

En cuanto al factor depresión también hubo resultados similares pero en menor impacto ya que a mayor deterioro cognitivo percibido se presentó un incremento moderado de la depresión, a mayor comentarios de otros; moderada-baja correlación con depresión a mayor capacidad cognitiva percibida; decremento en depresión 
y a mayor impacto en la calidad de vida; mayor incremento en la depresión, esto demuestra que el HADS fue estadísticamente significativo, aportando evidencia a la validez teórica del FACT COG ya quediversos estudios ${ }^{(17,18,20)}$ han reportado que la percepción de la función cognitiva en pacientes con tratamientos sistémicos de quimioterapia muestran sintomatología psicológica como ansiedad y depresión, además de que estas correlaciones serían mayores a las de instrumentos neuropsicológicos. Esto no quiere decir que la paciente no presente una alteración fisiológica de sus funciones cognitivas pues se ha planteado que esta percepción es una interpretación pesimista con sintomatología psicológica debido a un posible compromiso neuropsicológico ${ }^{(15)}$.

En este estudio también se comprobó que las correlaciones entre el FACT-COG y el Mini Examen el Estado Mental fueron bajas, ya que tanto la puntuación total del FACT-COG como sus 4 factores mostraron puntajes bajos que se encuentran entre 0,4 y 0,5 , es decir que a mayor deterioro cognitivo percibido, comentarios de otros, capacidades cognitivas percibidas e impacto en la calidad de vida, habrá correlación baja con el verdadero funcionamiento cognitivo de la paciente, el que un instrumento neuropsicológico tenga bajas correlaciones con el auto reporte de las funciones cognitivas de una persona según Wagner et al. ${ }^{(19)}$ se debe a que el auto informe hace que la paciente haga más consiente la disminución de la naturaleza de los síntomas además de la demanda de la persona día a día en sus actividades diarias, también se plantea que la evaluación neuropsicológica se realiza en ambientes controlados para obtener una puntuación objetiva mientras tanto la evaluación de la percepción del paciente se toma en cuenta en sus actividades de la vida diaria y como la percibe en esas situaciones, en el FACT-COG original la medición de rendimiento neuropsicológico obtuvo correlaciones de $0,4-0,5$ similar a las puntuaciones obtenidas en este estudio que oscilaban entre 0,4 y 0,5 .

El modelo contiene 4 factores con fuerte asociación entre ellos, tal como lo mostró el análisis exploratorio, lo que permite recomendar a este instrumento para evaluar función cognitiva percibida en pacientes con cáncer de mama. Los más poderosos indicadores del ajuste de la estructura del modelo (CFI, RMR y RMSEA) apuntan a que es un modelo equilibrado y parsimonioso ${ }^{(20)}$

El FACT-COG es un instrumento muy utilizado a nivel mundial, ya que ha sido validado en diferentes poblaciones, al comparar el presente estudio con las de otros países se puede observar que son muy similares tanto en su confiabilidad, cargas factoriales, varianza explicada y validez.

\section{Conclusiones}

El instrumento de Evaluación de Funcionalidad en Tratamiento para el Cáncer - Función Cognitiva (FACT-COG) es un instrumento que demostró adecuadas propiedades psicométricas en población oncológica mexicana por lo que aporta evidencia de la validez del instrumento, evaluada a través de las principales variables de correlación reportadas en la literatura científica y que fueron confirmados en este estudio.

Además de que el FACT-COG puede ser utilizado en población mexicana oncológica tanto en estudios clínicos y diagnósticos, para detectar específicamente la percepción de la paciente ante su función cognitiva derivado de su tratamiento 
oncológico y su impacto en la calidad de vida. Así mismo permitiría realizar referencia de los pacientes a la intervención clínica correspondiente de manera oportuna para mejorar la calidad de la atención recibida por parte del equipo multidisciplinario de salud, mejorando la adherencia al tratamiento y seguimiento; con el fin de que un especialista pueda realizar una evaluación objetiva y determinar si se requiere rehabilitación oportuna.

Se recomienda utilizar para futuras investigaciones clínicas, debido a que las pacientes con cáncer de mama pueden percibir que presentan efectos secundarios como alteraciones cognitivas que impactan en su calidad de vida y se pueden asociar al desarrollo de sintomatología de ansiedad, depresión, malestar emocional y fatiga.

\section{Referencias bibliográficas}

1. Organización Mundial de la Salud. Cáncer de mama: prevención y control. Factores de riesgo del cáncer de mama. 2017 [Acceso el 10 de septiembre de 2019]. Disponible en: http://www.who.int/topics/cancer/breastcancer/es/index1.html.

2. Globocan. Global Cancer Observatory, 2018. [Acceso el 10 de septiembre de 2019]. Disponible en: https:/gco.iarc.fr/today/data/factsheets/populations/484-mexico-fact-sheets.pdf

3. Ramalho M, Fontes F, Ruano L, Pereira S, Lunet N. Cognitive impairment in the first year after breast cancer diagnosis: a prospective cohort study. Breast 2017; 32:173-8. doi: 10.1016/j.breast.2017.01.018

4. Hermelink K, Voigt V, Kaste J, Neufeld F2, Wuerstlein R2, Bühner M, et al. Elucidating pretreatment cognitive impairment in breast cancer patients: the impact of cancer-related posttraumatic stress. J Natl Cancer Inst 2015;107:(7). doi: /10.1093/jnci/djv099

5. Cerulla N, Arcusa À, Navarro JB, de la Osa N, Garolera M, Enero C, et al. Cognitive impairment following chemotherapy for breast cancer: The impact of practice effect on results. J Clin Exper neuropsychol 2019;41:290-9. doi: 10.1080/13803395.2018.1546381

6. López-Santiago S, Cruzado JA, Feliu J. Daños neuropsicológicos asociados a los tratamientos quimioterapéuticos: Una propuesta de evaluación. Clínica Salud 2012; 23:3-24.

7. Cheung YT, Ng T, Shwe M, Ho HK, Foo KM, Cham MT, et al. Association of proinflammatory cytokines and chemotherapy-associated cognitive impairment in breast cancer patients: a multi-centered, prospective, cohort study. Ann Oncol 2015;26:1446-51. doi: $10.1093 /$ annonc/mdv206

8. Cano M, Díaz A, Fernández E, García C, Gutiérrez G, Menéndez A, Velasco T, Vena C. Quimioterapia: Guía para pacientes. Oviedo: Servicio de salud del principado de Asturias, 2009

9. Hermelink K, Untch M, Lux MP, Kreienberg R, Beck T, Bauerfeind I, et al. Cognitive function during neoadjuvant chemotherapy for breast cancer: results of a prospective, multicenter, longitudinal study. Cancer 2007;109:1905-13. doi: 10.1002/cncr.22610

10. Hermelink K, Buhner M, Sckopke P, Neufeld F, Kaste J, Voigt V, et al. Chemotherapy and post-traumatic stress in the causation of cognitive dysfunction in breast Cancer patients. J Natl Cancer Inst 2017;109:djx057. doi: 10.1093/jnci/djx057

11. van Dam FS, Boogerd W, Schagen SB, Muller MJ, Droogleever Fortuyn ME, Wall EV, Rodenhuis S. Impairment of cognitive function in women receiving adjuvant treatment for high-risk breast cancer: high-dose versus standard-dose chemotherapy. JNCI: J Natl Cancer Inst 1998;90:210-8. doi: 10.1093/jnci/90.3.210 
12. Shilling V, Jenkins V. Self-reported cognitive problems in women receiving adjuvant therapy for breast cancer. Eur J Oncol Nurs 2007;11: 6-15. doi: 10.1016/j.ejon.2006.02.005

13. Andreotti C, Root JC, Schagen SB, McDonald BC, Saykin AJ, Atkinson TM, et al. Reliable change in neuropsychological assessment of breast cancer survivors. Psychooncology 2016;25: 43-50. doi: 10.1002/pon.3799

14. Kohli S, Griggs JJ, Roscoe JA, Jean-Pierre P, Bole C, Mustian KM, et al. Self-reported cognitive impairment in patients with cancer. J Oncol Pract 2007;3: 54-9. doi: 10.1200/ JOP.0722001.

15. Biglia N, Bounous VE, Malabaila A, Palmisano D, Torta DME, d'Alonzo M, ... \& Torta R. Objective and self-reported cognitive dysfunction in breast cancer women treated with chemotherapy: a prospective study. Eur J Cancer Care 2012;21:485-92. doi: 10.1111/ j.1365-2354

16. Skirbe H, Hohn G, Klein P, Juliano M, Winell J, Rosenwald V, et al. Prevalence of selfreported cognitive dysfunction in breast cancer patients. J Clin Oncol 2013;2:131-131. doi: 10.1200/jco.2013.31.26_suppl.131.

17. Joly F, Rigal O, Noal S, Giffard B. Cognitive dysfunction and cancer: which consequences in terms of disease management? Psychooncology 2011;20:1251-8. doi: 10.1002/ pon. 1903

18. Bottino SMB, Garcia CPG, Viana BDM, Bottino CMDC. Depressive symptoms and cognitive deficits in a cancer patient submitted to chemotherapy with 5-Fluoracil: A case report. Dement Neuropsychol 2013;7:308-11. doi: 10.1590/S1980-57642013DN70300013

19. Wagner LI, Sweet J, Butt Z, Lai JS, Cella D. Measuring patient self-reported cognitive function: development of the functional assessment of cancer therapy-cognitive function instrument. J Support Oncol 2009; 7: W32-W39.

20. Cheung YT, Lim SR, Shwe M, Tan YP, Chan A. Psychometric properties and measurement equivalence of the English and Chinese versions of the functional assessment of cancer therapy-cognitive in Asian patients with breast cancer. Value Health 2013;16:1001-13. doi: $10.1016 /$ j.jval.2013.06.017

21. Joly F, Lange M, Rigal O, Correia H, Giffard F, Beaumont J, et al. French versión of the Functional Assessment of Cancer Therapy-Cognitive Function (FACT-Cog) versión 3. Support Care Cancer 2012;20:3297-305. doi: 10.1007/s00520-012-1439-2.

22. Park JH, Bae SH, Jung YS, Jung YM. The psychometric properties of the Korean version of the functional assessment of cancer therapy-cognitive (FACT-Cog) in Korean patients with breast cancer. Support Care Cancer 2015;23: 2695-703. doi: 10.1007/s00520-0152632-x

23. Folstein M, Folstein S, McHugh P. "Mini-Mental State": A practical method for grading the cognitive state of patients for the clinician. J Psychiatric Res 1975;12:189-98.

24. Reyes A, Beaman P, García-Peña C, Villa M, Heres J, Córdova A, et al. Validation of a modified version of the Mini-Mental State Examination (MMSE) in Spanish. Aging. Neuropsychol Cognition 2004;11: 1-11. doi: 10.1076/anec.11.1.1.29366

25.Zigmond A, Snaith R. The hospital anxiety and depression scale. Acta psychiatrica scandinavica 1983;67: 361-70.

26. Galindo Vázquez O, Benjet C, Juárez García F, Rojas Castillo E, Riveros Rosas A, Aguilar Ponce JL, et al. Propiedades psicométricas de la Escala Hospitalaria de Ansiedad y Depresión (HADS) en una población de pacientes oncológicos mexicanos. Salud mental 2015;38:253-8. doi: 10.17711/sm.0185-3325.2015.035

27. Ullman JB. Structural equation modeling: reviewing the basics and moving forward. J Pers Assess 2006; 87:35-50. 
28. Kline R. Principles and practice of structural equation modeling. 2nd. ed. New York: Guilford, 2005.

29. George D, Mailery M. Using SPSS for Windows step by step: a simple guide and reference.. Boston: Aliyn \& Bacon, 2001. [Acceso 10 de septeimbre de 2019]. Disponible en: http://www.gbv.de/dms/tib-ub-hannover/634302485.pdf

30. Byrne B. Structural equation modeling with AMOS: Basic concepts, applications, and programming, 2nd. New York: Routledge, 2010

31. Hu Lt, Bentler M. Fit Indices in covariance structure modeling: Sensitivity to underparameterized model misspecification. Psychol Methods 1998; 3: 424-53. doi: 10.1037/1082-989X.3.4.424 